\title{
Nanocurcumin-pyrroloquinoline formulation prevents hypertrophy-induced pathological damage by relieving mitochondrial stress in cardiomyocytes under hypoxic conditions
}

\author{
Sarita Nehra ${ }^{1}$, Varun Bhardwaj ${ }^{1}$, Anju Bansal ${ }^{1}$, Pronobesh Chattopadhyay ${ }^{2}$ and Deepika Saraswat ${ }^{1}$
}

This study investigates the therapeutic effect of a nanocurcumin formulation (NCF) containing nanocurcumin (NC) and pyrroloquinoline quinone $(P Q Q)$ on ameliorating hypoxia-induced stress in hypertrophied primary human ventricular cardiomyocytes (HVCM) under hypoxic conditions, as validated in a Sprague-Dawley rat model of chronic hypobaric hypoxia (cHH)-induced right ventricular hypertrophy (RVH). Based on our previous findings, here, we analyzed the improvement in the protective efficacy of NCF against mitochondrial damage. The electron transport chain Complexes' activities were analyzed as a chief operational center for mitochondrial homeostasis, along with key gene and protein markers for mitochondrial biogenesis, redox function, fatty acid oxidation, bio-energetic deficit and cell survival. NCF supplementation imparts cyto-protection from hypoxia-induced hypertrophy and damage in both in vitro and in vivo models while maintaining mitochondrial homeostasis better than NC and PQQ alone. This study proposes the use of NCF as a potential candidate molecule for imparting protection from high altitude-induced maladies in ascendants.

Experimental \& Molecular Medicine (2017) 49, e404; doi:10.1038/emm.2017.199; published online 1 December 2017

\section{INTRODUCTION}

Prolonged hypoxia-induced hypertrophy can cause pathological damage in cardiomyocytes that appear in the form of impaired mitochondrial homeostasis. ${ }^{1-4}$ The optimal functioning of mitochondria is tightly regulated by transcriptional control of the nuclear genome, which encodes mitochondrial regulatory proteins, including those required for operating electron transport chain (e.t.c.) Complexes I-V. ${ }^{5}$ However, the functions of these key regulatory proteins and Complexes are compromised under stress and cause cyto-damage by promoting excessive free radical leakage (Complexes I and III), ${ }^{6}$ metabolic imbalance (Complexes II and IV) ${ }^{7}$ and bioenergetic deficit (Complex V). ${ }^{8}$ Studies have shown close associations of impaired e.t.c. Complexes in cardiovascular damage. ${ }^{2,9}$ However, whether hypoxic stress promotes damage to mitochondria in hypertrophied cardiomyocytes remains unclear.

Multiple molecular pathways are involved in the modulation of cardiac hypertrophy. Akt/Gsk-3 $\beta$-mediated signaling remains the key modulatory pathway, among others. Akt and Gsk-3 $\beta$ are positive and negative regulators of cardiac hypertrophy, respectively. ${ }^{10}$ Akt phosphorylation and activation is directly involved in the modulation of cardiac hypertrophy by controlling downstream signaling cascades. ${ }^{11}$ In contrast, phosphorylation of Gsk-3 $\beta$ promotes its deactivation and further modulates hypertrophy. ${ }^{12}$ Studies have shown that Akt phosphorylation and activation promote dephosphorylation of Gsk-3 $\beta$, which in turn promotes cardiac hypertrophy. Many studies have shown that the stress-induced

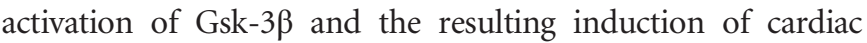
hypertrophy are deleterious. Further, Gsk-3 $\beta$ activation is directly involved in the regulation of primary transcriptional regulators of hypertrophy, that is, GATA- $4 .{ }^{13}$ Similarly, NFkB remains an important redox-sensitive transcriptional regulator that is required to maintain a variety of cellular functions, such as cell growth, survival and inflammation. ${ }^{14}$ The canonical pathway of NFkB signaling (NFkB-p65/RelA) has been well elucidated as a potential regulator of cardiomyocyte

\footnotetext{
${ }^{1}$ Experimental Biology Division, Department of Experimental Biology, Defence Institute of Physiology and Allied Science, Defence Research and Development Organization, Timarpur, New Delhi, India and ${ }^{2}$ Defence Research Laboratory, Tezpur, Assam, India

Correspondence: Dr D Saraswat, Experimental Biology Division, Department of Experimental Biology, Defence Institute of Physiology and Allied Science, Defence Research and Development Organization, Lucknow Road, Timarpur, New Delhi 110054, India.

E-mail: deepika_saras@yahoo.com

Received 28 October 2016; revised 11 April 2017; accepted 8 May 2017
} 
hypertrophy. ${ }^{15}$ Studies have shown that NFkB expression is essential for promoting hypertrophic growth in cardiomyocytes ${ }^{16}$ Knock-out studies relying upon deletion of functions of either functional units or inhibitors of NFKB stabilizers have shown to be lethal in embryonic developmental and can modulate cardiac dysfunction under stress. ${ }^{17,18}$ In addition to agonists, ligands and endotoxin-mediated onset of $\mathrm{NF \kappa B}$ signaling, mitochondrial reactive oxygen species (ROS) are also crucially involved in cellular pathology via the activation of NFKB by regulating programmed cell death. ${ }^{19-21}$ However, the association between hypoxia-induced NFKB activation and its potential effect on mitochondrial homeostasis in hypertrophied cardiomyocytes remains unknown.

Use of natural bio-active compounds promotes homeostasis under stress with minimal undesirable effects. In this regard, we have previously shown that nanotized curcumin (NC) ameliorates hypoxia stress both in vitro and in vivo.,3,22,23 Hypoxic stress elicits multiple physiological damages on normal physiological functions. ${ }^{24}$ Additionally, the normal functioning of cardiomyocytes critically relies upon an optimal oxygen supply. Thus, there remains a need for highly potent bio-active therapeutic compounds to abolish hypoxia-induced impairments. Therefore, we hypothesized that a combination of therapeutic bio-active compounds might demonstrate a potential strategy for reducing hypoxia-induced multi-faceted pathological damage. Pyrroloquinoline quinone (PQQ), a natural poly-phenolic, redox-factor and anti-oxidant compound of high nutritional value, is a potential candidate for improving hypoxia-induced damage in cardiomyocytes. ${ }^{25} \mathrm{PQQ}$ can regulate biological functions, ${ }^{26}$ including mitochondrial biogenesis, ${ }^{27}$ redox functions ${ }^{28,29}$ and reproductive health. ${ }^{30}$ Thus, to further improve the therapeutic properties of $\mathrm{NC}$, we propose the use of a nanocurcumin formulation (NCF) consisting of NC and PQQ together as a potential candidate molecule to ameliorate hypoxia-induced stress in hypertrophied cardiomyocytes.

\section{MATERIALS AND METHODS}

Ultra-pure, molecular grade chemicals, including PQQ (D7783), were procured from Sigma Aldrich (St Louis, MO, USA) or as otherwise stated. Antibodies were purchased from SantaCruz Biotechnology, Inc., Dallas, TX, USA, or as otherwise stated. Nanocurcumin (average particle size $200 \mathrm{~nm}$, zeta potential $-131 \mathrm{mV}$ ) was obtained as a kind gift from Professor Santosh Kar (KIIT University, Odisha, India).

\section{Study design}

The study was designed to prepare, characterize and assess improvements in the protective efficacy of NCF in in vitro and in vivo models of hypoxia-induced hypertrophy. Here we analyzed the effects of hypoxic stress on changes in mitochondrial regulators of homeostasis and its effects on the functions of e.t.c. Complexes. We also assessed the effect of NFKB activation on the molecular regulation of hypoxiainduced hypertrophy. Experimental animals and cell culture studies were performed in eight experimental groups, that is, normoxia vehicle controls (NVC), normoxia plus nanocurcumin $(\mathrm{N}+\mathrm{NC})$, normoxia plus PQQ $(\mathrm{N}+\mathrm{PQQ})$, normoxia plus nanocurcumin formulation $(\mathrm{N}+\mathrm{NCF})$, hypoxia vehicle controls (HVC), hypoxia plus nanocurcumin $(\mathrm{H}+\mathrm{NC})$, hypoxia plus PQQ $(\mathrm{H}+\mathrm{PQQ})$ and hypoxia plus nanocurcumin formulation $(\mathrm{H}+\mathrm{NCF})$. Sterile, neutral phosphatebuffered saline (PBS) was used as vehicle. All results were compared to NC- or PQQ-treated animals to compare improvements in the therapeutic potential of NCF under hypoxic conditions.

\section{Preparation and characterization of NCF}

A detailed description of the preparation and characterization of NCF can be found in the Supplementary Material.

\section{Cell culture, hypoxia exposure and protein isolation}

HVCM cells were maintained under the conditions of normoxia or hypoxia as previously described. ${ }^{3}$ Nuclear and cytoplasmic protein extracts and protein estimations were prepared according to the method described previously. ${ }^{4}$

\section{In vitro uptake and toxicity analysis of $\mathrm{NCF}$}

NCF was suspended in sterile PBS, and the suspension was stabilized via ultra-sonication for $15 \mathrm{~min}$ at $4{ }^{\circ} \mathrm{C}$ (Sonics Vibra Cell, Sonic and Materials, Inc., Newtown, CT, USA, pulse cycle $9 \mathrm{~s}$, amplitude $40 \%$ ). The cellular uptake of NCF was evaluated according to the method described by Kunwar et al. ${ }^{31}$ Briefly, the HVCM cells were grown to confluence in 96-well black plates and incubated with $100 \mu \mathrm{M}$ of NCF for $24 \mathrm{~h}$. The culture medium was removed, and cells were washed twice with PBS. Spectro-fluorometric analysis (FLUOStar Omega, BMG Labtech, Ortenberg, Germany) was performed to evaluate the cellular uptake of NCF (excitation wavelength/emission wavelength, that is, $\lambda_{\mathrm{ex}} / \lambda_{\mathrm{em}}$ read at $360 / 420 \mathrm{~nm}$ for NC and $360 / 460 \mathrm{~nm}$ for PQQ). An increase in NCF uptake was represented as a percentage change compared with normoxia vehicle control cells.

A detailed description of the in vitro cytotoxicity analysis can be found in the Supplementary Material.

\section{Cellular viability and hypertrophy}

A detailed description can be found in the Supplementary Material.

\section{Analysis of mitochondrial damage under hypoxic conditions in HVCM cells}

Hypoxia-induced changes in mitochondrial membrane potential $\left(\psi_{\mathrm{m}}\right)$ were analyzed as indicative of mitochondrial damage using a MitoLight Apoptosis Detection kit (APT242, Millipore, Billerica, MA, USA), and images were acquired $(\times 40)$ using a fluorescent microscope (IMAGER.M2, AxioCam MRc5, Carl Zeiss, Oberkochen, Germany). Further, cellular oxidative stress was analyzed in HVCM cells under hypoxic conditions for confirmation of cyto-damage. The mitochondrial redox balance was assessed by evaluating MnSOD (Manganesesuperoxide dismutase) activity and ROS leakage as previously described. ${ }^{4,32}$

\section{Immunocytofluorescence}

A detailed description can be found in the Supplementary Material.

\section{Experimental animals and ethics statement}

Age-matched adult male Sprague-Dawley rats $(220 \pm 10 \mathrm{~g})(6-8$ weeks $)$ were used for all of the experiments. The experimental animals were kept in the Institute's Experimental Animal Facility in clean cages and had equal access to water and standard chow (Lipton India Ltd., West Bengal, India), maintaining a normal $12 \mathrm{~h}$ light and dark cycle at $28 \pm 2{ }^{\circ} \mathrm{C}$ temperature and relative humidity $(55 \pm 2 \%)$. All experiments were approved by the Institutional Animal Ethical Committee 
in accordance with the Committee for Purpose of Control and Supervision of Experiments on Animals (27/1999/CPCSEA), Government of India.

\section{In vivo toxicity assessment}

Sub-acute toxicity in rats supplemented with $2000 \mathrm{mg} \mathrm{kg}^{-1}$ per day of NCF, p.o. for 28 days was performed by following the OECD guidelines. The animals were closely monitored for changes in body weight, fur texture and behavior and the development of skin infections. After completion of the experiment, the animals were killed by overdose of ketamine $\left(80 \mathrm{mg} \mathrm{kg}^{-1}\right.$ b.w.) and xylazine (10 $\mathrm{mg} \mathrm{kg}^{-1}$ b.w.) as previously described. ${ }^{23}$ Blood was withdrawn by left-ventricular puncture and was immediately subjected to bloodgas content analysis using an i-STAT analyzer (Abott, IL, USA) and hematological profiling (MS-4 Autoanalyzer, Melet Schloesing Laboratory, Osny, France). Post-drawing blood, animals were fixed in $4 \%$ neutral paraformaldehyde (PFA) for histopathological analyses of liver, heart and lungs.

Pharmacokinetic assessment of NCF in Sprague-Dawley rats A detailed description can be found in the Supplementary Material.

\section{Morphometry analysis and RVH}

Morphometry analysis of right ventricular hypertrophy (RVH) was performed by analyzing Fulton's index (RV/LV+S and RV/BW) and histopathological assessment via Masson's trichrome staining as previously described. ${ }^{22}$ Circulating levels of atrial natriuretic factor (ANF) (ab108797, Abcam, Cambridge, UK) and brain natriuretic peptide (BNP) (ab108816, Abcam) were estimated as markers of cardiac hypertrophy using commercially available ELISA kits; the assays were performed according to manufacturer's instructions. Tissue distribution of ANF was examined as a marker of hypertrophy by immunohistochemistry, and changes in tissue-architecture were analyzed by immunofluorescence staining using $\alpha$-actin/DAPI. Validation of $\mathrm{cHH}$-induced $\mathrm{RVH}$ was performed by analyzing the key molecular pathway for regulation of hypertrophy, that is, the Akt/Gsk$3 \beta$-mediated signaling cascade. ${ }^{33}$

Changes in pulmonary vascular architecture and morphometry post $\mathrm{cHH}$ exposure were made in PFA-fixed lung tissue by H\&E staining. Tissue sections were microscopically visualized for increments in pulmonary artery medial wall thickness and changes in pulmonary vascular architecture. ${ }^{34}$

\section{Isolation and functional analysis of cardiac mitochondria}

For evaluating damage to oxidative phosphorylation (OXPHOS) machinery, quantitative analyses of complete e.t.c. Complexes' activities were performed. Briefly, mitochondria were isolated from HVCM cells (Mitochondria Isolation Kit, 89879, Thermo Fisher, Waltham, MA, USA) and subjected to analysis of Complex I (AAMT001, Millipore), Complex II and III (ab109905, Abcam), Complex IV (ab109911, Abcam) and Complex V (AAMT005, Millipore) activities using commercially available kits.

For validating in vitro findings, mitochondria were isolated from right ventricles (Mitochondria Isolation Kit, 89801, Thermo Fisher) and were subjected to quantitative evaluation for changes in mitochondrial e.t.c. Complexes by western blot (Total OxPhos Complex Kit, 458099, Invitrogen, Waltham, MA, USA). Analysis of Complex I$\mathrm{V}$ activities was performed as mentioned above. Further, changes in gene and protein expression levels of key regulators of mitochondrial bio-genesis (mtTFA, Nrf1, Nrf2 and PGC1 $\alpha$ ), fatty acid metabolism
$(\operatorname{PPAR} \alpha / \beta / \gamma)$, redox-function (Nox-2, Cox-2), bio-energetic function (UCP-2, UCP-3) and cellular proliferation (Bcl2/Bax) were investigated via polymerase chain reaction (PCR) and western blot, respectively.

\section{Semi-quantitative polymerase chain reaction}

A detailed description can be found in the Supplementary Material and list of primers can be found in Supplementary Table T1.

\section{Immunoblot analysis}

A detailed description can be found in the Supplementary Material.

\section{Statistical analysis}

Data are expressed as the means \pm standard deviations (s.d.'s) for each experimental group performed in triplicate. The results were analyzed for statistical significance using one-way ANOVA. Values were considered to be statistically significant at ${ }^{\star} P \leqslant 0.05$ vs $\mathrm{NVC}$, ${ }^{* *} P \leqslant 0.01$ vs NVC, ${ }^{\#} P \leqslant 0.05$ vs HVC and ${ }^{\# \#} P \leqslant 0.01$ vs HVC. Nonsignificant changes are depicted as NS.

\section{RESULTS}

\section{Characterization of NCF}

The FTIR spectrum of NCF was expected to elucidate the complex appearance of variable peaks as characteristics of PQQ (containing multiple bonds including $\mathrm{N}-\mathrm{H}$ and $\mathrm{C}-\mathrm{N}$ groups) and signature peaks of curcumin.

The FTIR spectrum of NCF revealed distinct patterns of broad and sharp peaks ranging from 650 to $4000 \mathrm{~cm}^{-1}$. The appearance of distinctly sharp peaks at 648 and $1028 \mathrm{~cm}^{-1}$ depicted the presence of $\mathrm{C}-\mathrm{H}$ bends and $\mathrm{C}-\mathrm{N}$ stretches, respectively. ${ }^{35}$ A strong peak at $1280.7 \mathrm{~cm}^{-1}$ indicated a $\mathrm{C}-\mathrm{H}$ wag structure. ${ }^{36}$ The consecutive appearance of strong peaks at 1371.3 and $1431.1 \mathrm{~cm}^{-1}$ indicated stretching vibrations of $\mathrm{C}-\mathrm{H}_{3}$ and C-C bonds, respectively. The appearance of broad peaks at $1633.6 \mathrm{~cm}^{-1}$ and a strong peak at $1587 \mathrm{~cm}^{-1}$ depicted stretching vibrations of $\mathrm{C}-\mathrm{C}$ and $\mathrm{N}-\mathrm{H}$ bend structures. ${ }^{35}$ A broad peak ranging from 2800 to $3000 \mathrm{~cm}^{-1}$ depicted the presence of $\mathrm{H}-\mathrm{C}=\mathrm{O}: \mathrm{C}-\mathrm{H}$ bends along with $\mathrm{C}-\mathrm{H}$ stretches. Peaks in the range of $3500-3750 \mathrm{~cm}^{-1}$ appeared as characteristic for curcumin, with strong peaks at 3631 and $3745 \mathrm{~cm}^{-1}$, indicating vibrations of $\mathrm{O}-\mathrm{H}$ stretches in ring structures ${ }^{35}$ (Figure 1a).

The DLS analysis consistently depicted improvements in physico-chemical properties of NCF in terms of zeta potential, particle size, electrophoretic mobility and conductivity compared with NC. The zeta potential of NCF was $-50.3 \mathrm{mV}$ $(-30 \mathrm{mV}$ in $\mathrm{NC})$, with an average particle size $1955 \mathrm{~nm}$ (212 $\mathrm{nm}$ in NC). The electrophoretic mobility and conductivity of NCF were $-3.941 \mu \mathrm{m} \mathrm{cm} \mathrm{V}^{-1} \mathrm{~s}^{-1}\left(-2.348 \mu \mathrm{m} \mathrm{cm} \mathrm{V}^{-1} \mathrm{~s}^{-1}\right.$ in NC) and $0.404 \mathrm{mS} \mathrm{cm}^{-1}\left(0.0359 \mathrm{mS} \mathrm{cm}^{-1}\right.$ in NC), respectively (Table 1). Similarly, homogenous populations of NCF particles were visible in SEM and TEM images (Figure 1b). The DLS analysis certainly depicted negative zeta potential with efficient electrophoretic mobility and conductivity, which represent evidence of high NCF bio-stability. This finding was further corroborated with a pharmacokinetic assessment (Figure 1c) of NCF, indicating a high mean resident time, 

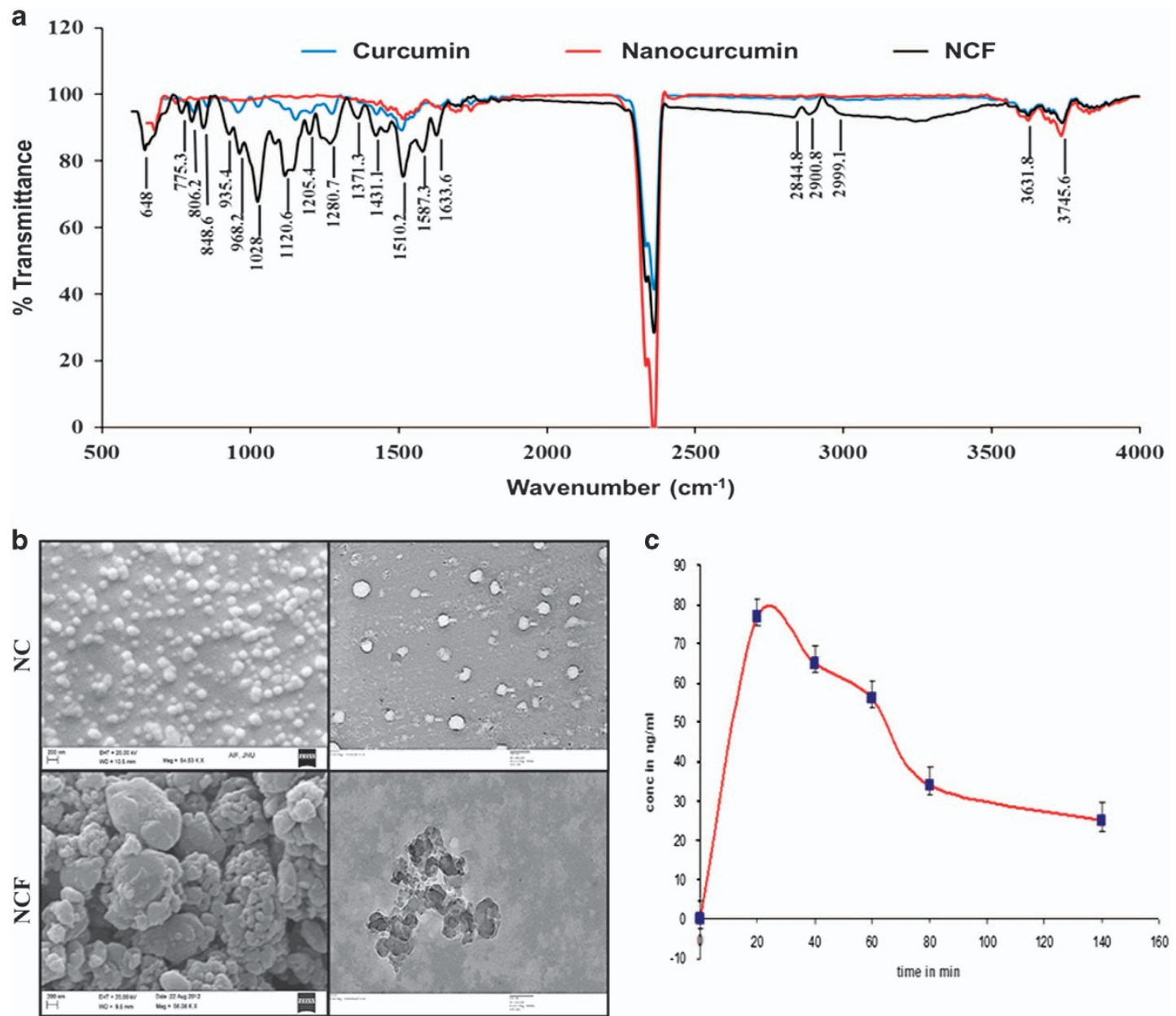

Figure 1 The FTIR analysis of nanocurcumin formulation (NCF) (a) along with SEM and TEM (b) and the plasma retention kinetics of NCF in rats (c).

Table 1 Changes in zeta potential, mobility, size and conductivity of NCF compared with NC by dynamic light scattering

\begin{tabular}{lcccc}
\hline Material & Zeta potential $(m V)($ mean \pm s.d.) & Mobility $\left(\mu m \mathrm{~cm} \mathrm{~V}^{-1} \mathrm{~s}^{-1}\right)($ mean \pm s.d.) & Size $(n m \pm$ s.d.) & Conductivity $\left(m S \mathrm{~cm}{ }^{-1}\right)($ mean \pm s.d.) \\
\hline NC & $-30 \pm 2.33$ & $-2.348 \pm 0.24$ & $212 \pm 221$ & $0.0359 \pm 0.0002$ \\
NCF & $-50.3 \pm 2.41$ & $-3.941 \pm 0.36$ & $1955 \pm 238$ & $0.404 \pm 0.001$ \\
\hline
\end{tabular}

Abbreviations: NC, nanocurcumin; NCF, nanocurcumin formulation.

Data are represented as the means \pm s.d. from three independent experiments.

clearly suggesting improved NCF bio-availability and biostability (Table 2).

\section{Toxicity analysis}

NCF was physiologically safe and did not cause any toxicity in HVCM cells. HVCM cells did not exhibit any evidence of micro-nuclei or DNA damage and maintained normal cellular division (Supplementary Figure S1). No significant changes in LDH activity or cellular viability were observed in HVCM cells (Supplementary Figure S1). Similarly, the physical appearances, body weight and behavioral tendencies and the blood gas contents and hematological parameters were normal in NCFsupplemented animals compared with control animals (Supplementary Tables T2-T4). No clinically relevant damage to vital organs (heart, lungs and liver) was observed in NCFsupplemented animals compared with normal animals (Supplementary Figure S2).

\section{NCF imparts cyto-protection under hypoxic conditions}

Hypoxia stress decreased HVCM cellular viability (decreased to $20 \%$ compared with NVC). Hypoxia-mediated cell death was confirmed using caspase-3, -7 activation by FACS and was further confirmed by TUNEL assay. Significant NCF uptake was observed in HVCM cells (Supplementary Figure S3) under hypoxic conditions. NCF treatment $\left(500 \mathrm{ng} \mathrm{ml}^{-1}\right)$ significantly improved cellular viability (97\%) compared with NC- and PQQ-treated cells under hypoxic conditions compared with HVC (76\% and 69\%, respectively). Corroborating these 
findings, decreased caspase- $3,-7$ activation and TUNEL positivity in NCF-treated cells further confirmed the enhancement in cellular viability under hypoxic conditions compared with NC- and PQQ-treated cells (Supplementary Figure S3).

\section{NCF protects from hypoxia-induced hypertrophy}

Induction of hypertrophy was evident in HVCM cells under hypoxic conditions, as indicated by morphometric analysis (by 35\%) and the increases in FITC-leucine (by 63\%) and phenyla-

Table 2 Qualitative data representing the pharmacokinetic profile of NCF

\begin{tabular}{|c|c|}
\hline Parameters & Results \\
\hline$k_{\mathrm{el}}$ & $0.017 \pm 0.007$ \\
\hline $\mathrm{AUC}_{0-t}\left(\mathrm{mg} \mathrm{ml}^{-1} \mathrm{~min}^{-1}\right)$ & $1.19 \pm 0.44$ \\
\hline$A \cup C_{0-\infty}\left(\mathrm{mg} \mathrm{ml}^{-1} \mathrm{~min}^{-1}\right)$ & $2.55 \pm 0.63$ \\
\hline$t_{1 / 2}(\min )$ & $28.55 \pm 18.11$ \\
\hline$V_{\mathrm{d}}\left(\mathrm{kg}^{-1}\right)$ & $40.11 \pm 13.29$ \\
\hline$C_{1}\left(\mathrm{~kg}^{-1} \mathrm{~min}^{-1}\right)$ & $84 \pm 27.27$ \\
\hline MRT (min) & $58.22 \pm 29.56$ \\
\hline$k_{\alpha}\left(I \min ^{-1}\right)$ & $0.22 \pm 0.10$ \\
\hline$C_{\max }\left(\mathrm{mg} \mathrm{ml}^{-1}\right)$ & $0.03 \pm 0.002$ \\
\hline$t_{\max }(\min )$ & $13.09 \pm 4.1$ \\
\hline
\end{tabular}

Abbreviations: AUC, area under curve; MRT, mean residence time; NC,

nanocurcumin; NCF, nanocurcumin formulation.

Data are represented as the means \pm s.d. from three independent experiments. lanine (by 65\%) uptake and was confirmed from the increases in ANF (by 85\%) and BNP (by 93\%) levels compared with the NVC group (Figure 2a-d). Significant declines in cell size (by 30\%) and FITC-leucine (by 23\%) and phenylalanine (by 24\%) uptake predicted decreased hypertrophy in NCF-supplemented cells under hypoxic conditions compared with the HVC group (Figure 2a-d). Importantly, PQQ treatment effectively improved cellular viability but failed to demonstrate anti-hypertrophic effects.

Similarly, cHH-induced $\mathrm{RVH}$ was evident in animals due to the more than four-fold increases in $\mathrm{RV} / \mathrm{LV}+\mathrm{S}$ and $\mathrm{RV} / \mathrm{BW}$ content (Figure 3b). Simultaneous increase collagen accumulation of both types and tissue ANF and $\alpha$-actin expression levels confirmed the hypertrophic growth, along with the increased gene expression levels of myocardial markers of matrix remodeling, that is, collagen type 1 (Colla1 and Col3a1) (up to 1.8 -fold) and 3 with matrix metallopeptidase (MMP) 2 and 9 (up to 3.3-fold) compared with normoxia control animals (Figure $3 \mathrm{c}-\mathrm{g}$ ). Increases in the circulating levels of ANF (2.3fold) and BNP (5.3-fold) confirmed $\mathrm{cHH}$-induced RVH in animals (Figure $3 \mathrm{a}$ and $\mathrm{c}$ ). Treatment of animals with NCF effectively reduced RV/LV+S (1.2-fold) and RV/BW (0.7-fold) contents, with parallel decreases in collagen accumulation, ANF expression and $\alpha$-actin levels. These findings were validated by further significant decreases in circulating levels of ANF (1.17fold) and BNP (1.63-fold) and the expression levels of Colla1 and Col3a1 (up to 1.6-fold) and MMPs (up to 3.1-fold) in

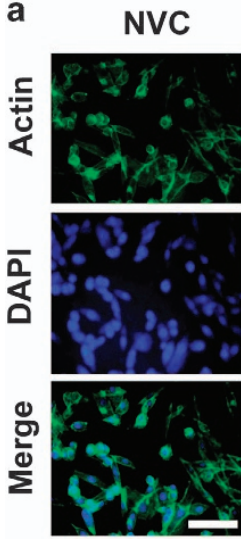

b

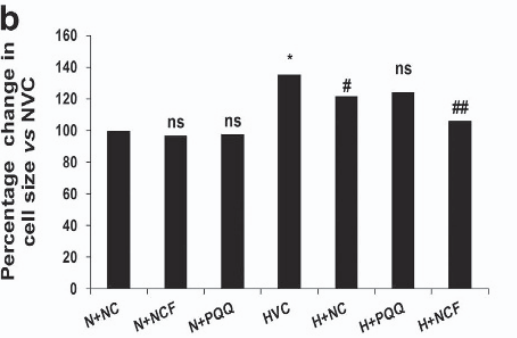

$\mathbf{N}+\mathbf{N C}$
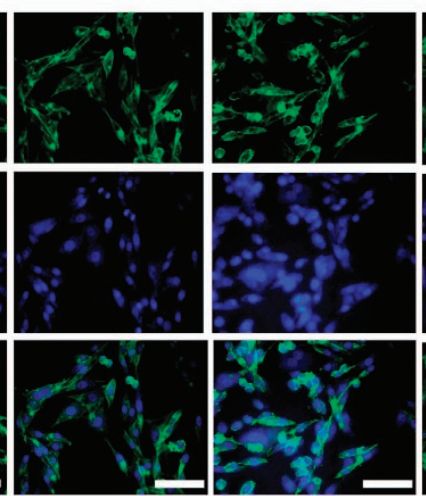

C

$\mathrm{N}+\mathrm{NCF}$
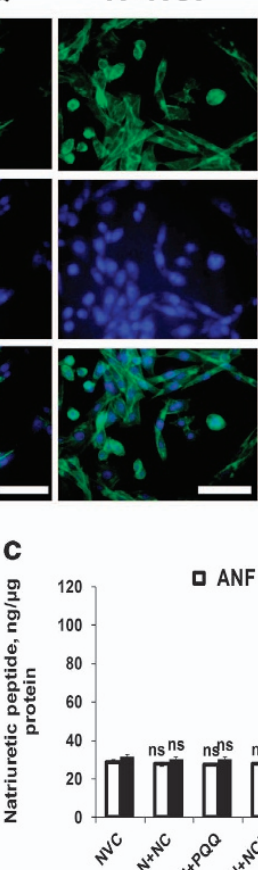

HVC
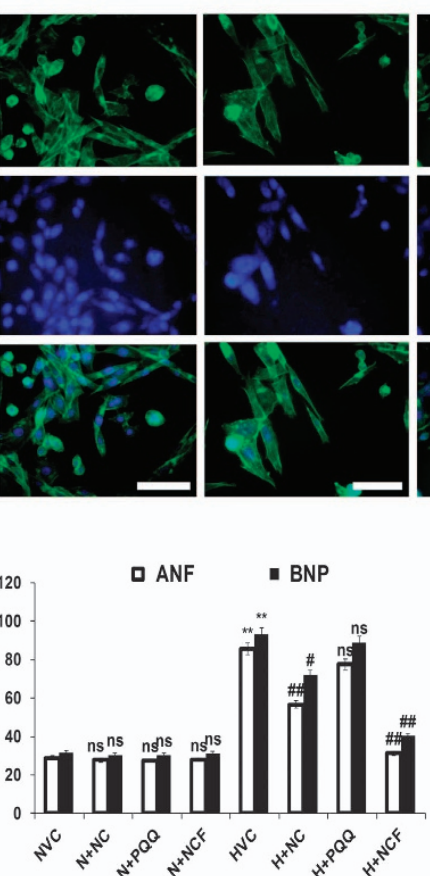

$\mathrm{H}+\mathrm{NC}$
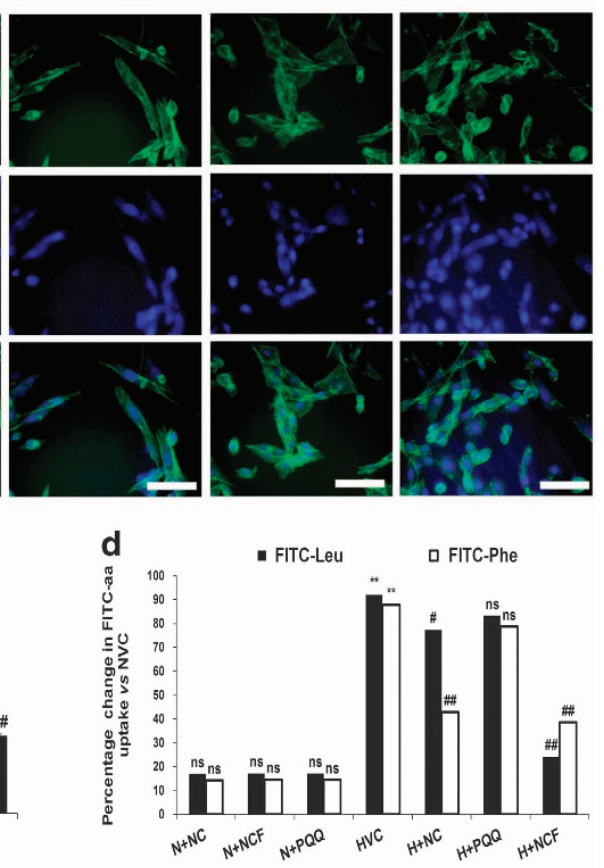

Figure 2 The effect of nanocurcumin formulation (NCF) on hypoxia-induced hypertrophy in human ventricular cardiomyocytes (HVCM) cells. Increments in cell size $(\times 40)(\mathbf{a}, \mathbf{b})$ along with atrial natriuretic factor (ANF) and brain natriuretic peptide (BNP) levels (c) depicted hypertrophic growth. Increments in FITC-leucine and FITC-phenylalanine uptake further confirmed hypoxia-induced hypertrophy (d). NCF supplementation effectively modulated hypoxia-induced hypertrophy in vitro. Data are expressed as the means \pm s.d. Values were considered to be statistically significant at ${ }^{*} P \leqslant 0.05$ vs NVC, ${ }^{* *} P \leqslant 0.01$ vs NVC, ${ }^{*} P \leqslant 0.05$ vs HVC and \#\# $P \leqslant 0.01$ vs HVC. Nonsignificant changes are depicted as NS. Scale bar, $20 \mu \mathrm{m}$. 


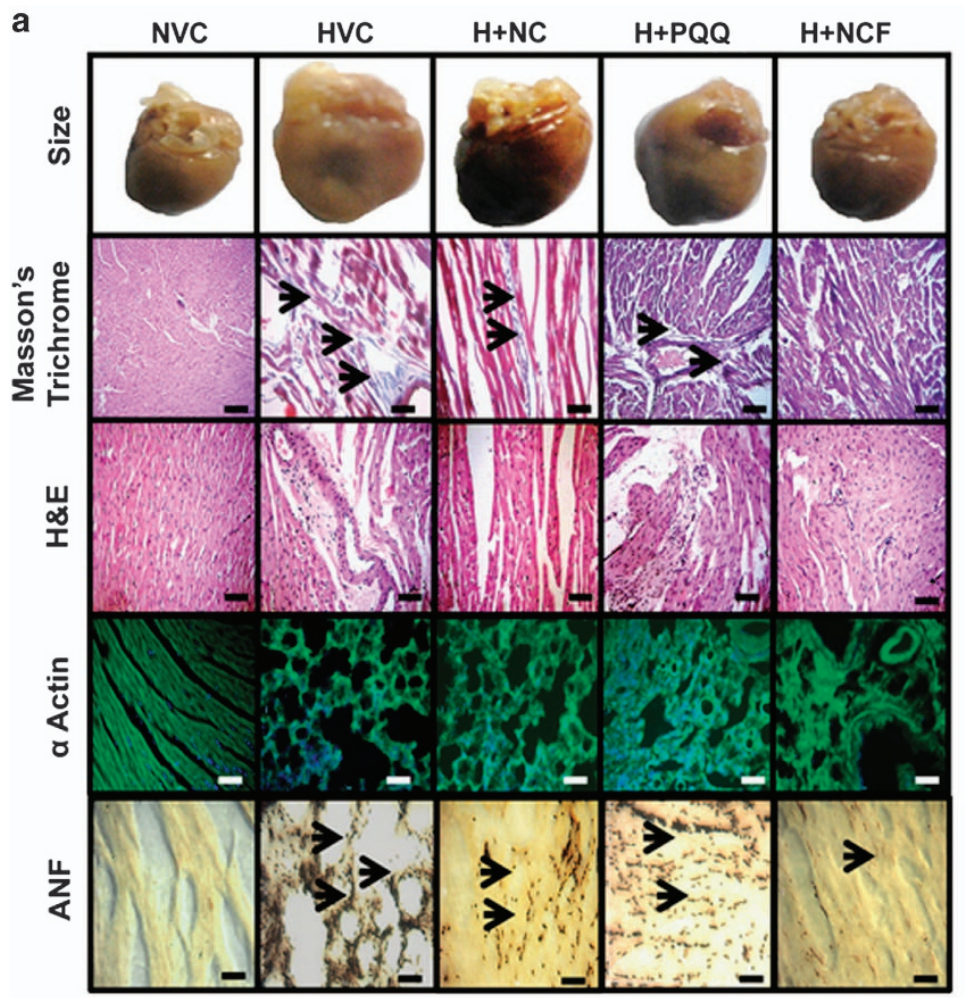

b
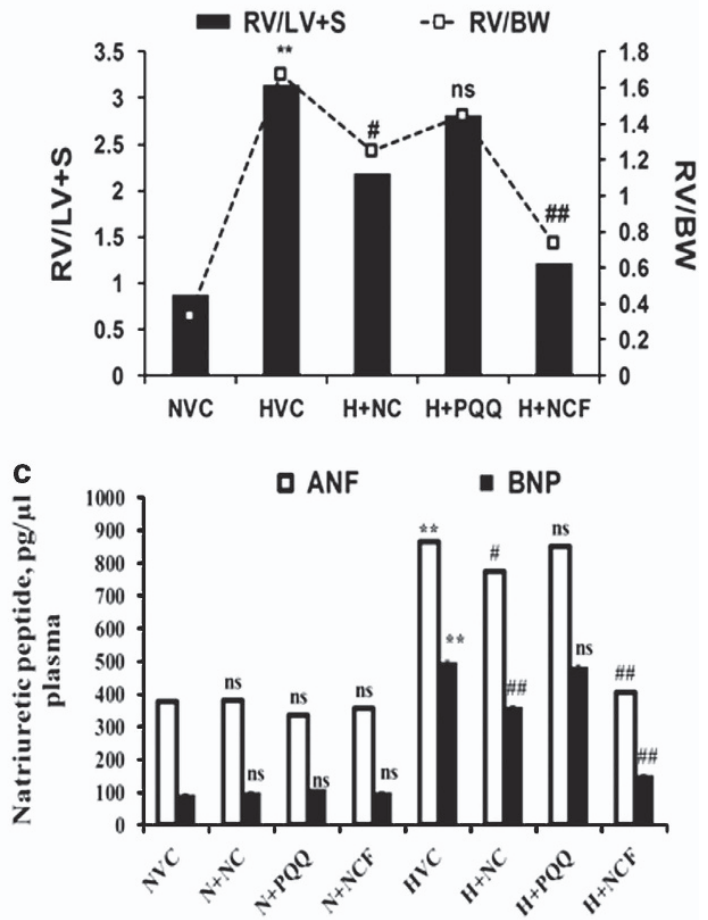

d
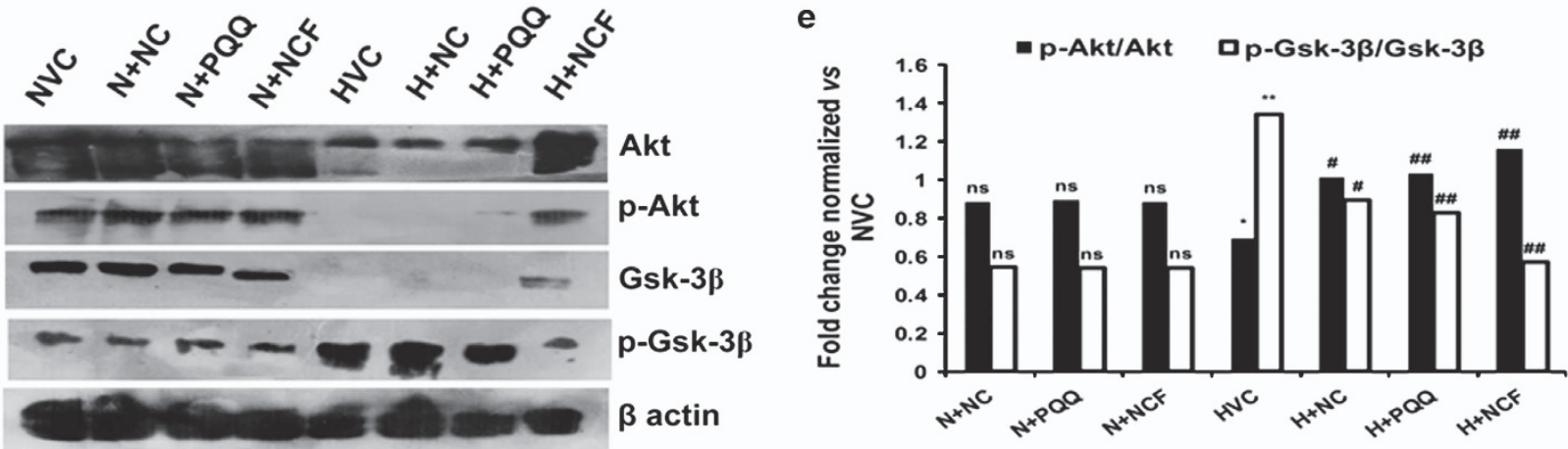

f
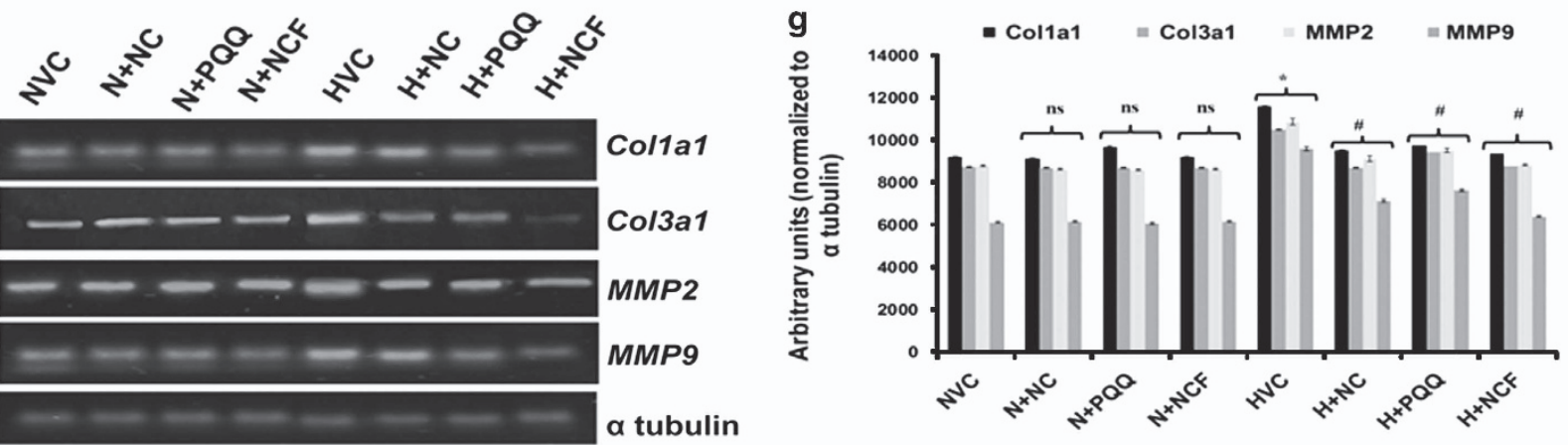

Figure 3 Representative figures showing the effect of nanocurcumin formulation (NCF) supplementation on $\mathrm{cHH}$-induced $\mathrm{RVH}$ in rats. Chronic HH-mediated RVH was evident in animals by increases in heart size, collagen accumulation (arrows), Fulton's index and histopathology staining $(\times 40)(\mathbf{a}, \mathbf{b})$. Tissue expression levels of skeletal $\alpha$-actin and atrial natriuretic factor (ANF) were increased due to hypertrophic growth (b). Circulating levels of ANF and brain natriuretic peptide (BNP) further confirmed $\mathrm{cHH}$-induced RVH in rats (c). Phosphorylation and activation of Akt-Gsk signaling promoted hypertrophic growth in rats under hypoxic conditions (d, e). Changes in gene expression levels of markers of myocardial matrix remodeling (Colla1 and Col3a1, along with MMP2 and 9) (f, g) in various experimental groups. Data are expressed as the means \pm s.d. NCF supplementation modulated chronic hypobaric hypoxia (cHH)-induced right ventricular hypertrophy $(\mathrm{RVH})$ better than nanocurcumin $(\mathrm{NC})$ and pyrroloquinoline quinone $(\mathrm{PQQ})$ treatments. Values were considered to be statistically significant at ${ }^{* *} P \leqslant 0.01$ vs NVC, ${ }^{\#} P \leqslant 0.05$ vs HVC and ${ }^{\#} P \leqslant 0.01$ vs HVC. Non-significant changes are depicted as NS. Scale bar, $10 \mu \mathrm{m}$. 

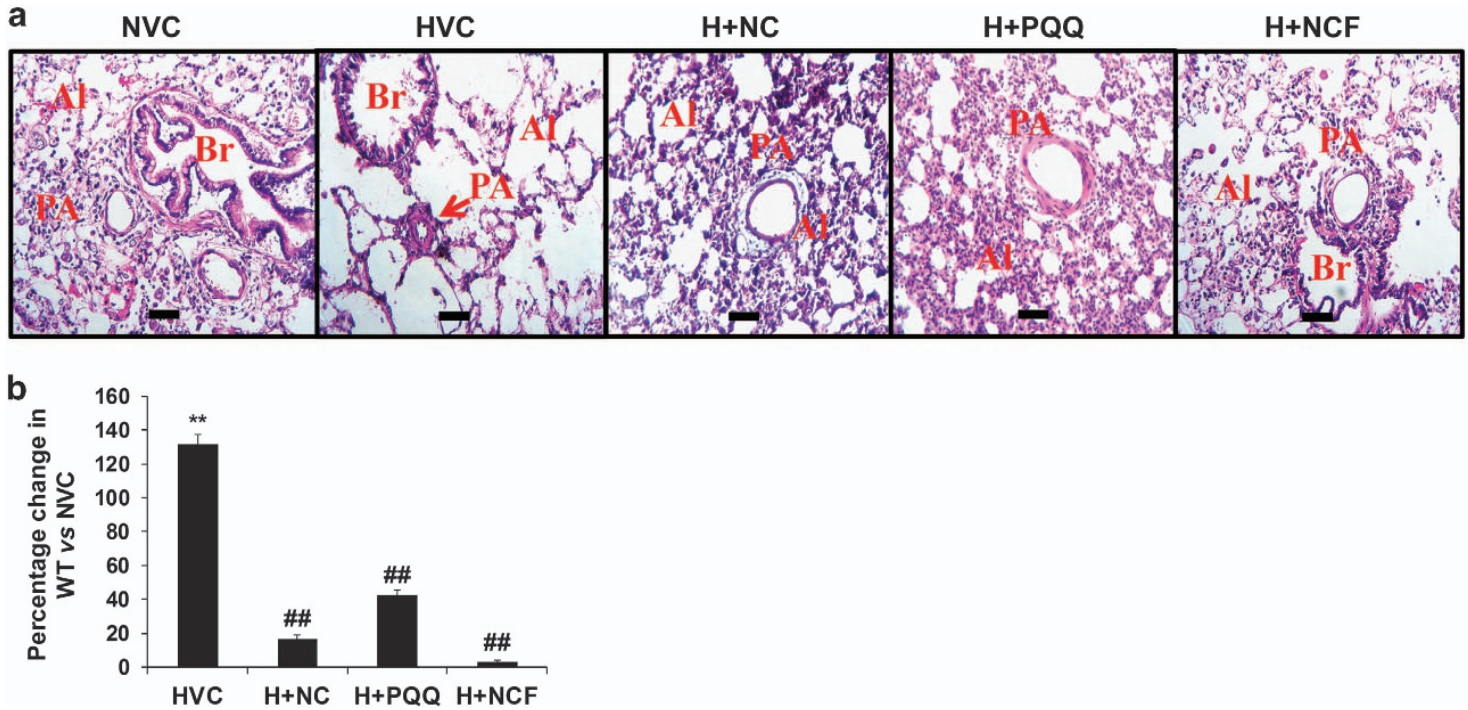

Figure 4 Figure showing changes in lung histology in various experimental groups (a), as evident by morphological changes in alveolar spaces $(\mathrm{Al}, \mathrm{red})$ and bronchioles $(\mathrm{Br}$, red). Histopathological staining $(\times 40)$ revealed increases in pulmonary arteries $(\mathrm{PA}$, red) and medial wall thickness (WT) in HVC groups, while nanocurcumin formulation (NCF) supplementation showed significant decreases compared with HVC, NC and PQQ (b). Data are expressed as the means \pm s.d. Values were considered to be statistically significant at $* * P \leqslant 0.01$ vs $N V C$ and ${ }^{\# \#} P \leqslant 0.01$ vs HVC. Scale bar: $10 \mu \mathrm{m}$.

NCF-supplemented animals compared with vehicle-treated animals under $\mathrm{cHH}$.

The improvement in NCF efficacy was further corroborated based on the Akt/Gsk-3 $\beta$-signaling pathway. $\mathrm{cHH}$ promoted p-Gsk-3 $\beta /$ Gsk-3 $\beta$ upregulation and downregulated p-Akt/Akt contents (Figure 3d and e). NCF supplementation showed significant restoration in the $\mathrm{p}$-Akt/Akt and $\mathrm{p}-\mathrm{Gsk}-3 \beta / \mathrm{Gsk}-3 \beta$ contents $(P \leqslant 0.05)$ compared with NC-treated cells under hypoxic conditions, clearly demonstrating the enhanced cardio-protective efficacy of NCF under $\mathrm{cHH}$.

\section{cHH-induced RVH in animals due to increased pulmonary resistance}

The pulmonary vasculature underwent discrete morphometric and architectural changes under $\mathrm{cHH}$. $\mathrm{H} \& \mathrm{E}$ staining in rat lungs revealed vascular remodeling of the pulmonary bed. The alveoli were scattered in appearance, fewer in number and larger in size (Figure 4a). The pulmonary arterial lumen appeared narrower, and the pulmonary artery (PA) medial wall underwent muscularization, thus promoting resistance to blood flow. Exposure to $\mathrm{cHH}$ led an increase in PA medial wall thickness (WT), which increased by $132 \% \quad(P \leqslant 0.01)$ in experimental animals compared with NVC (Figure 4). Importantly, animals supplemented with NCF showed a sharp decline in WT $(2.8 \%)(P \leqslant 0.01)$ and maintenance of the vascular architecture when compared with HVC (2.8\%) (Figure 4a and b). The WT declined to $16 \%$ and $42 \%$ in NC- and PQQsupplemented animals, respectively $(P \leqslant 0.01)$, when compared with HVC controls. These qualitative and quantitative data suggest that pulmonary vascular resistance increased under $\mathrm{cHH}$ due to narrowing of the PA. The results herein provide evidence that NCF supplementation effectively induced morphometric and functional modulation in pulmonary vasculature animals under $\mathrm{cHH}$ better than $\mathrm{NC}$ and PQQ alone.

\section{NCF protects from hypoxia-induced stress by maintaining mitochondrial function}

Hypoxic stress promoted damage to HVCM cells by disrupting the mitochondrial membrane potential $\left(\psi_{\mathrm{m}}\right)$, thus promoting mitochondrial damage-mediated apoptosis (Figure 5a and b). Excessive ROS leakage and decreased MnSOD activity further validated hypoxia-mediated mitochondrial damage to HVCM cells (Supplementary Figure S4). Further, exposure of HVCM cells and rodent hearts to $\mathrm{cHH}$ resulted in decreased activities of Complex I-V, depicting damage to mitochondrial chief functional units (Figure $5 \mathrm{c}-\mathrm{i}$ ). The data are in accordance with previously described damage to e.t.c. Complexes in rodent skeletal muscles. ${ }^{37}$ Accompanying these findings, we found that Nox-2 and Cox-2 expression levels increased under $\mathrm{cHH}$, suggesting compromised functions of Complexes I and IV, respectively (Figure 6a-d and Supplementary Figure S5A-H), along with decreased expression levels of e.t.c. Complexes in rodent hearts (Figure 6e and f). Decreased levels of PPAR $\alpha / \beta / \gamma$, mtTFA, Nrf1, Nrf2 and PGC1 $\alpha$ clearly depict impaired mitochondrial biogenesis, while decreased levels of UCP-2 and UCP-3 clearly demonstrate diminished bio-energetic efficacy due to the impaired function of Complex $\mathrm{V}$ under hypoxic conditions.

NCF treatment significantly restored e.t.c. Complexes' activity levels both in vitro and in vivo better than NC or PQQ alone (Figure $5 \mathrm{c}-\mathrm{g}$ ). Better regulation in $\psi_{\mathrm{m}}$ was evident in NCFtreated HVCM cells under hypoxic conditions compared with $\mathrm{NC}$ or PQQ or vehicle (Figure $5 \mathrm{a}$ and $\mathrm{b}$ ). Importantly, the markers of mitochondrial bio-genesis and functional control (PPAR $\alpha / \beta / \gamma$, mtTFA, Nrf1, Nrf2 and PGC1 $\alpha)$, along with bio- 

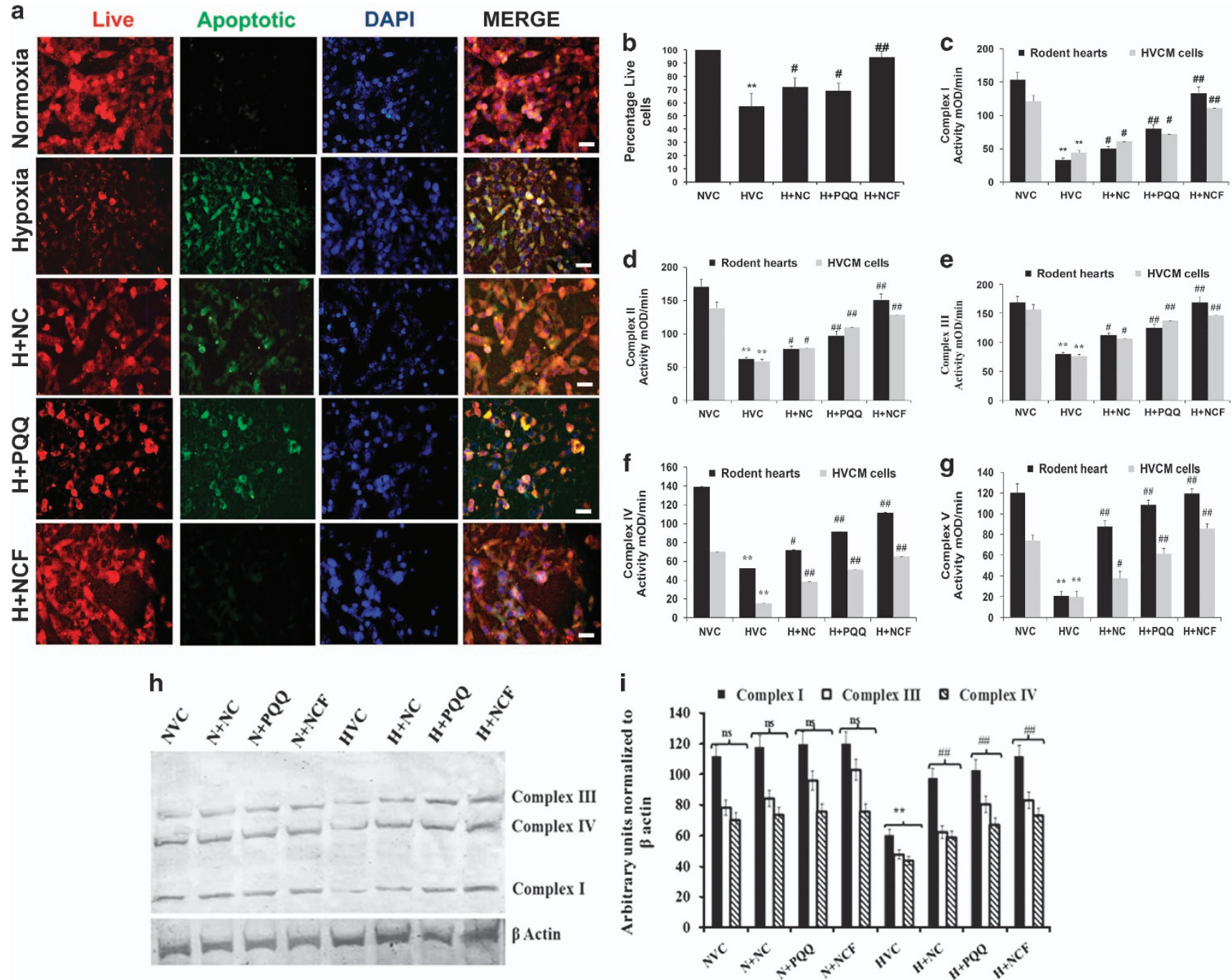

Figure 5 Figure showing changes in mitochondrial homeostasis under hypoxic conditions. Human ventricular cardiomyocytes (HVCM) cells experienced apoptotic cell death due to hypoxia-mediated impairments in membrane potential $(\times 40)(\mathbf{a}, \mathbf{b})$ depicting damage to mitochondrial homeostasis. Hypoxia-induced modulations in mitochondrial electron transport chain (e.t.c.) Complexes' activities (c-g) were also evident both in vitro and in vivo. (h, i) The effects of $\mathrm{cHH}$-induced modulation on regulators of oxidative phosphorylation. Data are

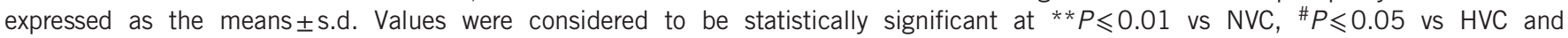
$\# \# \leqslant 0.01$ vs HVC. Non-significant changes are depicted as NS. Scale bar, $10 \mu \mathrm{m}$.

energetic efficiency (UCP-2,-3) and cell-survival (Bcl2, Bax), were more efficiently restored in NCF-treated animals than in those treated with $\mathrm{NC}$ and PQQ alone compared with vehicle under $\mathrm{cHH}$ (Figure 6a-d and Supplementary Figure S5A-H). NCF-supplemented animals exhibited restoration of the expression levels of Complexes I-V along with their activities better than either treatment alone (Figure $5 \mathrm{~h}$ and i). Collectively, these data demonstrate robust improvements in mitochondrial protective efficacy of NCF in cardiomyocytes compared with $\mathrm{NC}$ and $\mathrm{PQQ}$.

\section{NCF supplementation prevents NFKB-p65 activation under hypoxic conditions \\ The cellular activation and cytoplasmic accumulation of NFKB- p65 activation and cytoplasmic accumulation started as early as}

$6 \mathrm{~h}$ following the onset of hypoxia and reached their peaks by $24 \mathrm{~h}$ (Figure 7a-g and Supplementary Figures S6 and S7) in HVCM cells. However, nuclear translocation of NFkB-p65 was observed only in cells exposed to hypoxia for $24 \mathrm{~h}$ (Figure 7a-g).

Similarly, our in vivo findings confirmed NFkB activation in the right ventricles under $\mathrm{cHH}$ (Figure $7 \mathrm{f}$ and $\mathrm{g}$ and Supplementary Figure S8). To confirm the pathogenicity of cHH-induced RVH in rats, we compared NFkB tissue expression and changes in mitochondrial e.t.c. Complexes' activities along with histopathological changes in a pre-established model of monocrotaline-induced $\mathrm{RVH}^{38}$ Monocrotaline infusion promoted RVH, decreased e.t.c. Complex activities and increased NFkB expression in the right ventricles (Figure $8 \mathrm{a}-\mathrm{d})$. Together, these data clearly depict $\mathrm{cHH}$-induced $\mathrm{RVH}$ 


\section{Gene expression}
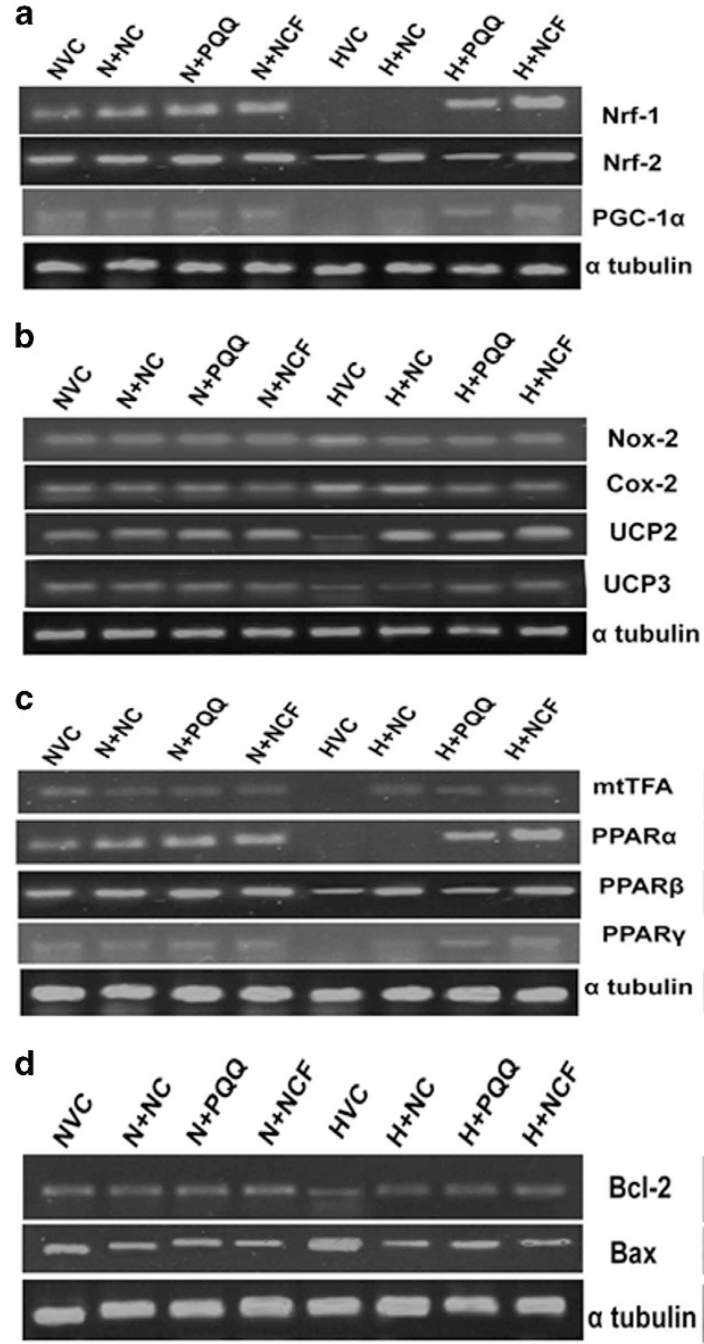

Nrf-1

Nrf-2

PGC-1a

a tubulin

Protein expression
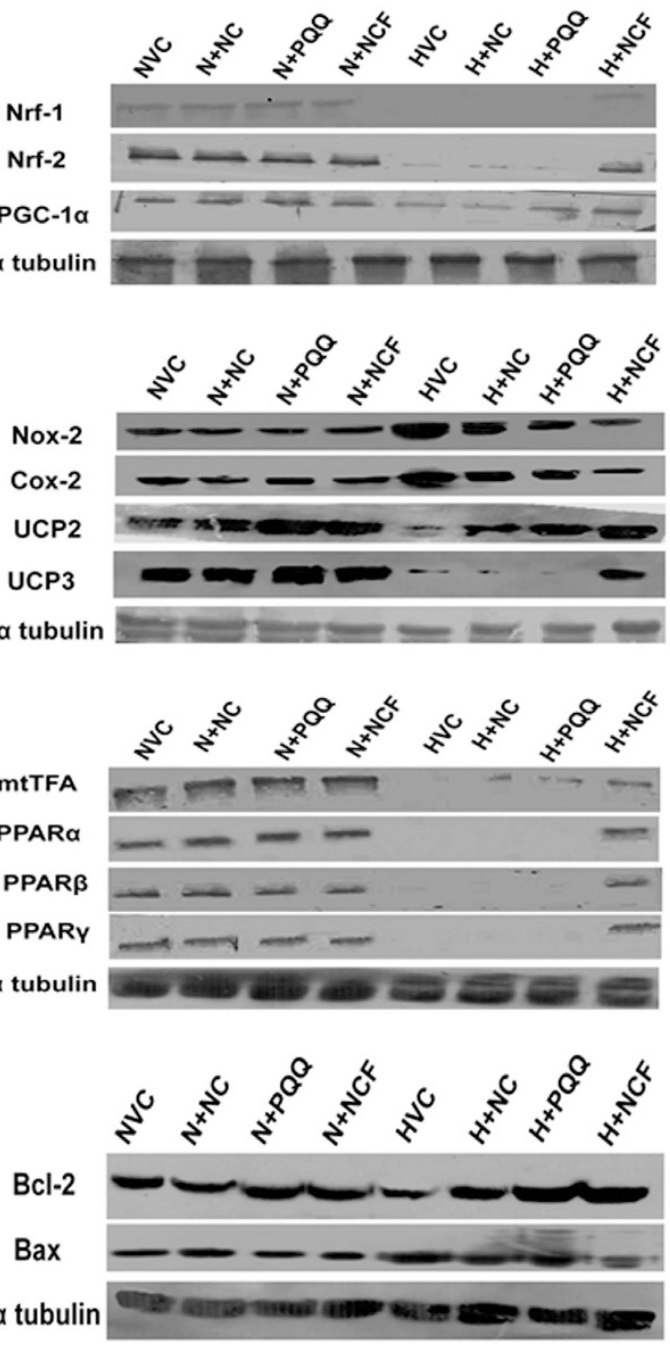

Figure 6 Figure showing gene and protein expression changes in mitochondrial homeostasis regulators: chronic hypobaric hypoxia (cHH)mediated decreases in regulators of mitochondrial biogenesis (mtTFA, Nrf1, Nrf2 and PGC1 $\alpha$ ) (a), redox function (Nox-2 and Cox-2) (b), fatty acid regulation (PPAR $\alpha / \beta / \gamma)$ (c) and cell survival (Bcl2, Bax) (d) were evident in both gene and protein expression studies. Values were considered to be statistically significant at ${ }^{*} P \leqslant 0.01$ vs NVC, ${ }^{\#} P \leqslant 0.05$ vs HVC and ${ }^{\#} P \leqslant 0.01$ vs HVC. Non-significant changes are depicted as NS.

and damage-mediated pathogenicity in rat hearts similar to a previously established model of monocrotaline-induced RVH and failure. ${ }^{39}$

NCF treatment resulted in significant decreases in cytoplasmic and nuclear levels of NFkB-p65 compared with vehicle/ NC/PQQ-treated cells and animals (Figure $7 \mathrm{a}-\mathrm{f}$ ), but $\mathrm{NC}$ and PQQ treatments failed. Collectively, the data demonstrate that cHH-mediated RVH and cardiomyocyte damage are dependent on NFKB-p65 activation, similar to monocrotaline-induced RVH (Figure $8 \mathrm{~b}$ and $\mathrm{c}$ ).

\section{DISCUSSION}

Cardiac hypertrophy appears as an initial adaptive response to hypoxia, but prolonged stress induces de-compensation and irreversible damage. The increase in systemic oxygen demand under hypoxic stress is well elucidated. ${ }^{24}$ To meet this increased demand for oxygenated blood, the heart frequently pumps more blood toward the lungs to improve systemic oxygenation. The heart undergoes hypertrophy under this sustained workload, with special relevance to the right ventricle. ${ }^{40}$ However, chronic hypoxic stress also promotes a decline in the oxygenation capacity of the lungs under chronic hypoxic stress due to excessive fluid retention, which causes an increase in the pulmonary resistance to the blood flowing from the right ventricles. However, to ensure an uninterrupted systemic blood supply, the hypertrophied right ventricle continuously pumps blood towards the lungs. Under this state of increased volume and pressure overload, the right side of the heart eventually undergoes de-compensation and suffers from pathological damage. ${ }^{40,41}$ Herein, we observed an increase in the pulmonary 
a
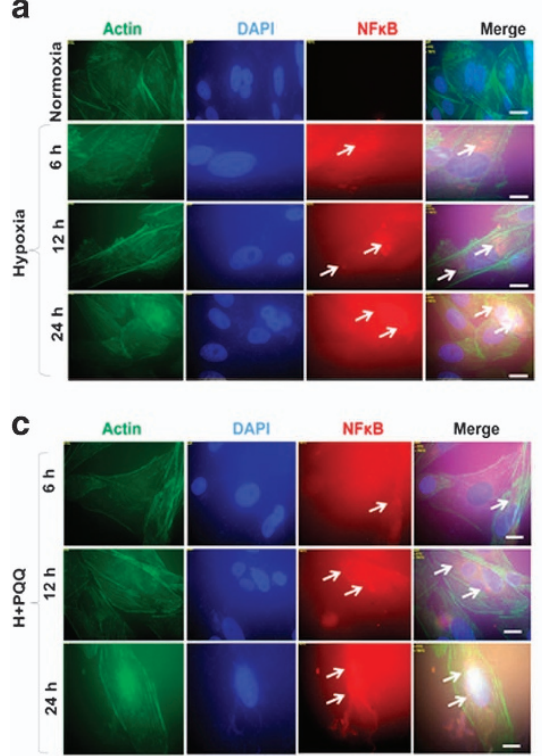

b

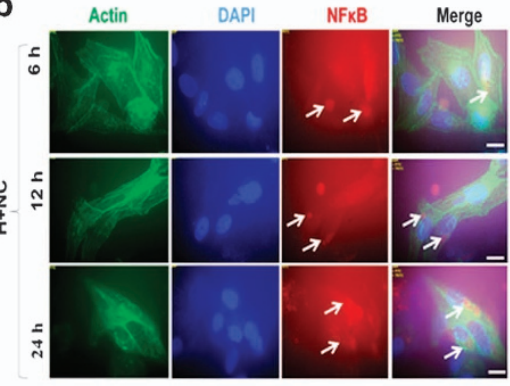

d
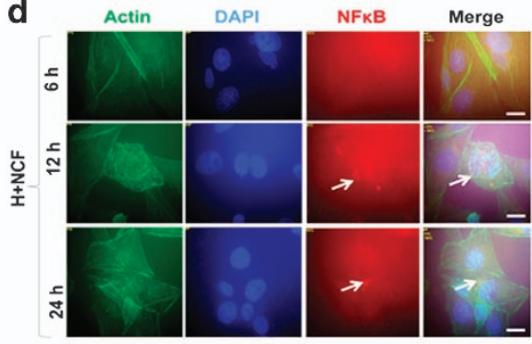

e $\mathrm{HVC} \quad \mathrm{H}+\mathrm{NC} \quad \mathrm{H}+\mathrm{PQQ} \quad \mathrm{H}+\mathrm{NCF}$ Nuclear NVC 6h 12h 24h6h 12h 24h 6h 12h 24h 6h 12h 24h NFKB - - - -

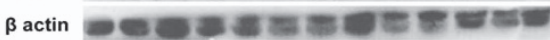

\section{$\mathbf{f}$}

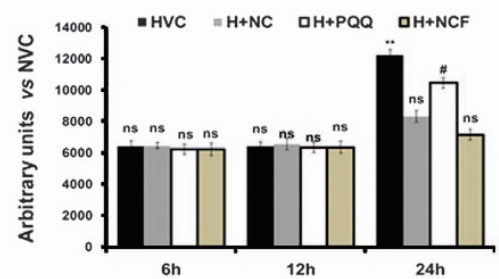

g

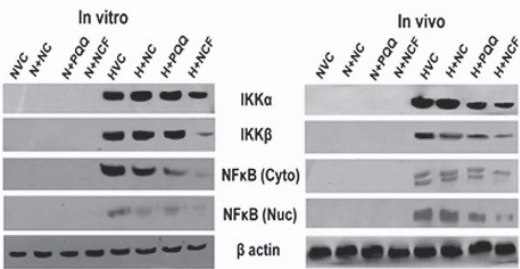

Figure 7 Representative figure showing NFKB activation under hypoxic conditions: human ventricular cardiomyocytes (HVCM) cells experienced cytoplasmic NFKB activation as early as $6 \mathrm{~h}$ following the onset of hypoxia $(\times 100)(\mathbf{a}-\mathbf{d})$, while nuclear translocation was observed only at $24 \mathrm{~h}$ following onset (e, f). Rodent right ventricles and HVCM cells demonstrated NFKB activation under chronic HH or hypoxic exposure for $24 \mathrm{~h}$, respectively (g). Supplementation of HVCM cells and rats with nanocurcumin formulation (NCF) resulted in markedly decreased NFKB activation, followed by NC and PQQ alone. Scale bar, $20 \mu \mathrm{m}$.
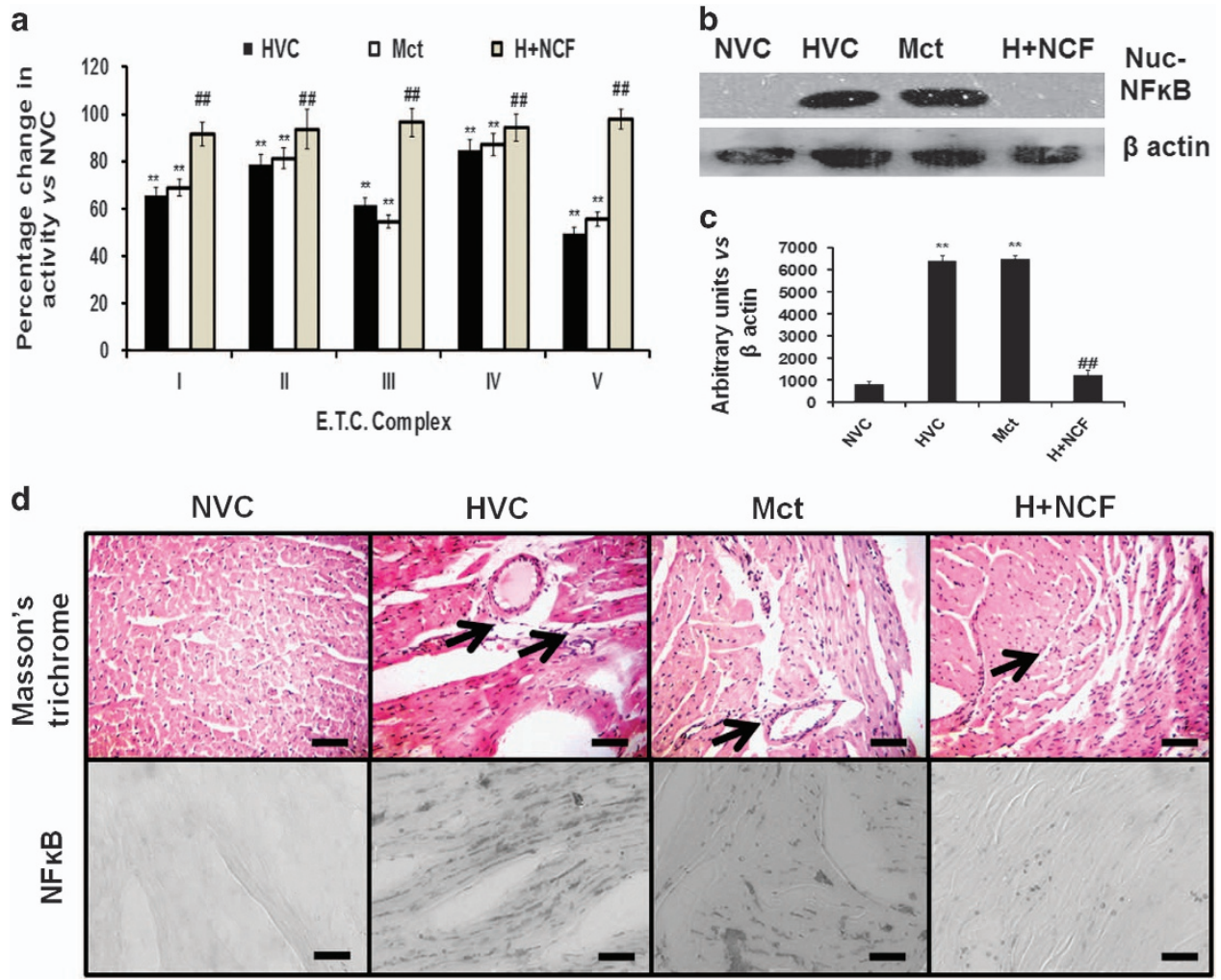

Figure 8 The comparative pathogenicity in chronic $\mathrm{HH}$ - and monocrotaline-induced RVH models: Chronic HH- and monocrotaline (Mct)induced RVH promoted increases in mitochondrial electron transport chain (e.t.c.). Complexes' I-V activity levels (a). Both models showed NFKB nuclear accumulation and tissue expression, along with increased tissue collagen accumulation $(\times 40)($ arrows) (b-d). Data are expressed as the means \pm s.d. Values were considered to be statistically significant at $* * P \leqslant 0.01$ vs NVC and \#\# $P \leqslant 0.01$ vs HVC. Scale bar, $10 \mu \mathrm{m}$. 
artery medial wall thickness and a decrease in the pulmonary artery lumen, which promotes an increase in the pulmonary resistance to blood flow. These changes might have led to sustained pressure and volume overload on the right ventricle, which eventually stimulated de-compensation in the chamber. ${ }^{40,41}$ Thus, the data in the present study provide evidence that the heart undergoes pathological damage due to de-compensation arising in the right ventricle that is secondary to the increased pulmonary vascular resistance. However, increased pulmonary resistance is directly associated with pulmonary hypertension; further investigations are needed to clarify the potential effects of cHH-induced hemodynamic changes on pathological RVH. ${ }^{40,41}$

In the present study, we found that severe damage to Complexes I-V clearly demonstrated that hypertrophied cardiomyocytes underwent mitochondrial stress under hypoxic conditions, both in vitro and in vivo. Previous studies have shown that stress-induced decreases in the functions of these Complexes are inseparably associated with cardiomyopathies and heart failure. ${ }^{2,9,42,43}$ However, to the best of our knowledge, this study provides the first report that chronic hypoxic stress promoted mitochondrial stress by impairing e.t.c. Complex activities in hypertrophied cardiomyocytes. Since optimal cardiomyocyte functioning relies on a constant supply of the optimum amount of oxygen, hypoxia-mediated oxidative stress might have contributed to such severe damage to the e.t.c. complex activities. The smooth flow of electrons through all five Complexes determines optimal cardiomyocyte function. ${ }^{44}$ As hypoxic stress promotes severe oxidative damage, excessive free-radical leakage-mediated redox imbalances might have initiated the mitochondrial damaging events that further led to impairments in the e.t.c. Complex activities. Further, chronic hypoxic stress impaired not only mitochondrial regulators of cell-survival, redox-maintenance and metabolism, but severe damage to regulators of mitochondrial bio-genesis was also evident. Decreased expression levels of the e.t.c. Complexes along with their activities clearly demonstrate that impaired mitochondrial homeostasis remains a critical molecular event behind $\mathrm{cHH}$-induced de-compensatory $\mathrm{RVH}$ and damage.

Further molecular investigation suggested that NFkB-p65 activation emerged as a potential regulator of hypoxia-induced hypertrophy, both in vitro and in vivo. Importantly, in vitro activation of NFKB-p65 and stabilization of IKK $\alpha / \beta$ were observed as early as $6 \mathrm{~h}$ following the onset of hypoxia. These data and our previous report strongly suggest that hypoxiamediated onset of hypertrophy and activation of NFkB-p65 in HVCM cells seems to be synchronous events $(6 \mathrm{~h}) .^{3}$ To ensure the role of NFkB-p65 activation in our animal model of RVH, we compared our findings with a previously established model of monocrotaline-induced RVH. Interestingly, monocrotalineinduced RVH was accompanied by NFkB-p65 activation and damage to e.t.c. Complex machinery, similar to our model of cHH-induced RVH. ${ }^{45}$ These findings suggest that the pathological events arising in the $\mathrm{cHH}$-induced model of $\mathrm{RVH}$ show pre-clinical similarity to a drug-induced model of RVH. However, since monocrotaline-induced RVH remains secondary to pulmonary hypertension, it is important to assess quantitative hemodynamic changes in pulmonary arterial pressure in a cHH-induced model of RVH. Collectively, the present study shows that hypoxic stress was sufficient to induce NFKB nuclear translocation and to initiate the transcriptional activation of downstream signaling genes, thus promoting hypertrophy and damage in rats. These damages appeared in the form of de-compensated RVH along with mitochondrial damage and demonstrate the transition of cardiomyocyte hypertrophy from an adaptive physiological state to a maladaptive pathological state.

This information and synchronous molecular events suggest that chronic hypoxia-mediated de-compensatory RVH appeared in animals in response to excessive pressure and volume overload, secondary to enhanced pulmonary vascular resistance. This de-compensation mediated pathological damage in the right heart by impairing e.t.c. Complexes. These data suggest that although stress-induced activation of NFKB is necessary for onset of hypertrophy, chronic stress-mediated sustained NFkB upregulation might have promoted decompensation and pathological damage. ${ }^{16}$ However, the exact molecular events behind NFK-mediated pathology require further investigations.

NCF supplementation was highly effective in modulating hypoxia-induced hypertrophy compared with NC and PQQ alone, clearly depicting an improvement in the cyto-protective efficacy of NCF. Importantly, PQQ treatment showed an improvement in cellular viability under hypoxic conditions but failed to modulate hypertrophy. These findings suggest that though oxidative stress plays a crucial role in the pathogenicity of cardiac hypertrophy, other molecular events might collectively play key regulatory roles in promoting pathogenicity under hypoxic conditions. Similarly, NC supplementation showed modulations of hypertrophy and pathology but did not impart sufficient therapeutic regulation of mitochondrial health under hypoxic conditions. Together, these findings emphasize the inference that NCF supplementation could provide comprehensive therapeutic benefits under stress. NCF supplementation imparted simultaneous protection from $\mathrm{cHH}$-induced damage to pulmonary architecture and damage to the right side of the heart. Robust improvements in e.t.c. Complex expression and activity levels with NCF treatment clearly demonstrate improvements in the therapeutic and cytoprotective potentials of NCF under hypoxic conditions compared with NC or PQQ alone. The data collectively suggest the potential efficacy of combinatorial therapeutics to combat multi-factorial pathologies mediated by hypoxic stress and further strengthen our hypothesis that combinatorial therapeutics might be an effective solution to hypoxia-mediated clinical problems.

\section{CONFLICT OF INTEREST}

The authors declare no conflict of interest. 


\section{ACKNOWLEDGEMENTS}

The authors sincerely thank the Director, Defence Institute of Physiology and Allied Sciences for providing necessary facilitation to perform the present research work.

\section{PUBLISHER'S NOTE}

Springer Nature remains neutral with regard to jurisdictional claims in published maps and institutional affiliations.

1 Essop MF. Cardiac metabolic adaptations in response to chronic hypoxia. J Physiol 2007; 584: 715-726.

2 Choksi KB, Boylston WH, Rabek JP, Widger WR, Papaconstantinou J. Oxidatively damaged proteins of heart mitochondrial electron transport complexes. Biochim Biophys Acta 2004; 1688: 95-101.

3 Nehra S, Bhardwaj V, Ganju L, Saraswat D. Nanocurcumin prevents hypoxia induced stress in primary human ventricular cardiomyocytes by maintaining mitochondrial homeostasis. PLOS ONE 2015; 10: e0139121.

4 Nehra S, Bhardwaj V, Kalra N, Ganju L, Bansal A, Saxena S et al. Nanocurcumin protects cardiomyoblasts $\mathrm{H} 9 \mathrm{c} 2$ from hypoxia-induced hypertrophy and apoptosis by improving oxidative balance. J Physiol Biochem 2015; 71: 239-251.

5 Heilbronn LK, Gan SK, Turner N, Campbell LV, Chisholm DJ. Markers of mitochondrial biogenesis and metabolism are lower in overweight and obese insulin-resistant subjects. J Clin Endocrinol Metab 2007; 92: 1467-1473.

6 Powell CS, Jackson RM. Mitochondrial complex I, aconitase, and succinate dehydrogenase during hypoxia-reoxygenation: modulation of enzyme activities by MnSOD. Am J Physiol Lung Cell Mol Physiol 2003; 285: L189-L198.

7 Sorescu D, Griendling KK. Reactive oxygen species, mitochondria, and $\mathrm{NAD}(\mathrm{P}) \mathrm{H}$ oxidases in the development and progression of heart failure. Congest Heart Fail 2002; 8: 132-140.

8 Ventura-Clapier R, Garnier A, Veksler V. Transcriptional control of mitochondrial biogenesis: The central role of PGC-1 $\alpha$. Cardiovasc Res 2008; 79: 208-217.

9 Redout EM, Wagner MJ, Zuidwijk MJ, Boer C, Musters RJP, van Hardeveld $\mathrm{C}$ et al. Right-ventricular failure is associated with increased mitochondrial complex II activity and production of reactive oxygen species. Cardiovasc Res 2007; 75: 770-781.

10 Hardt SE, Sadoshima J. Negative regulators of cardiac hypertrophy. Cardiovasc Res 2004; 63: 500-509.

11 Morisco C, Zebrowski D, Condorelli G, Tsichlis P, Vatner SF, Sadoshima J. The Akt-glycogen synthase kinase 3beta pathway regulates transcription of atrial natriuretic factor induced by beta-adrenergic receptor stimulation in cardiac myocytes. J Biol Chem 2000; 275: 14466-14475.

12 Haq S, Choukroun G, Kang ZB, Ranu H, Matsui T, Rosenzweig A et al. Glycogen synthase kinase-3beta is a negative regulator of cardiomyocyte hypertrophy. J Cell Biol 2000; 151: 117-130.

13 Morisco C, Seta K, Hardt SE, Lee Y, Vatner SF, Sadoshima J. Glycogen synthase kinase 3beta regulates GATA4 in cardiac myocytes. J Biol Chem 2001; 276: 28586-28597.

14 Gordon JW, Shaw Ja, Kirshenbaum LA. Multiple facets of NF-kB in the heart: to be or not to NF-кB. Circ Res 2011; 108: 1122-1132.

15 Hirotani S, Otsu K, Nishida K, Higuchi Y, Morita T, Nakayama $\mathrm{H}$ et al. Involvement of nuclear factor-kappaB and apoptosis signal-regulating kinase 1 in G-protein-coupled receptor agonist-induced cardiomyocyte hypertrophy. Circulation 2002; 105: 509-515.

16 Purcell NH, Tang G, Yu C, Mercurio F, DiDonato JA, Lin A. Activation of NFkappa $B$ is required for hypertrophic growth of primary rat neonatal ventricular cardiomyocytes. Proc Natl Acad Sci USA 2001; 98: 6668-6673.

17 Timmers L, van Keulen K, Hoefer IE, Meijs MFL, van Middelaar B, den Ouden $\mathrm{K}$ et al. Targeted deletion of NF-kappaB P50 enhances cardiac remodeling and dysfunction following myocardial infarction. Circ Res 2009; 104: 699-706.

$18 \mathrm{Beg}$ AA, Baltimore D. An essential role for NF-kappaB in preventing TNF-alpha-induced cell death. Science 1996; 274: 782-784.
19 Quillet-Mary A, Jaffrézou JP, Mansat V, Bordier C, Naval J, Laurent G. Implication of mitochondrial hydrogen peroxide generation in ceramideinduced apoptosis. J Biol Chem 1997; 272: 21388-21395.

20 Sabri A, Hughie HH, Lucchesi PA. Regulation of hypertrophic and apoptotic signaling pathways by reactive oxygen species in cardiac myocytes. Antioxid Redox Signal 2003; 5: 731-740.

21 Fritah A, Steel JH, Nichol D, Parker N, Williams S, Price A et al. Elevated expression of the metabolic regulator receptor-interacting protein 140 results in cardiac hypertrophy and impaired cardiac function. Cardiovasc Res 2010; 86: 443-451.

22 Nehra S, Bhardwaj V, Kar S, Saraswat D. Chronic hypobaric hypoxia induces right ventricular hypertrophy and apoptosis in rats: therapeutic potential of nanocurcumin in improving adaptation. High Alt Med Biol 2016; 17: 342-352.

23 Nehra S, Bhardwaj V, Bansal A, Saraswat D. Nanocurcumin accords protection against acute hypobaric hypoxia induced lung injury in rats. $J$ Physiol Biochem 2016; 72: 763-779.

24 Giordano FJ. Oxygen, oxidative stress, hypoxia, and heart failure. J clinincal Investig 2005; 115: 500-5008.

25 Killgore J, Smidt C, Duich L, Romero-Chapman N, Tinker D, Reiser K et al. Nutritional importance of pyrroloquinoline quinone. Science 1989; 245: 850-852.

26 Rucker R, Chowanadisai W. Potential physiological importance of pyrroloquinoline quinone. Altern Med Rev 2009; 14: 268-277.

27 Stites T, Storms D, Bauerly K, Mah J, Harris C, Fascetti A et al. Pyrroloquinoline quinone modulates mitochondrial quantity and function in mice. J Nutr 2006; 136: 390-396.

28 Chowanadisai W, Bauerly KA, Tchaparian E, Wong A, Cortopassi GA, Rucker RB. Pyrroloquinoline quinone stimulates mitochondrial biogenesis through CAMP response element-binding protein phosphorylation and increased PGC-1alpha expression. J Biol Chem 2010; 285: $142-152$.

29 Harris CB, Chowanadisai W, Mishchuk DO, Satre MA, Slupsky CM, Rucker RB. Dietary pyrroloquinoline quinone (PQQ) alters indicators of inflammation and mitochondrial-related metabolism in human subjects. J Nutr Biochem 2013; 24: 2076-2084.

30 Steinberg F, Stites TE, Anderson P, Storms D, Chan I, Eghbali S et al. Pyrroloquinoline quinone improves growth and reproductive performance in mice fed chemically defined diets. Exp Biol Med 2003; 228: 160-166.

31 Kunwar A, Barik A, Mishra B, Rathinasamy K, Pandey R, Priyadarsini KI. Quantitative cellular uptake, localization and cytotoxicity of curcumin in normal and tumor cells. Biochim Biophys Acta 2008; 1780: 673-679.

32 Nehra S, Bhardwaj V, Saraswat D. Amlodipine protects rat ventricular cardiomyoblast $\mathrm{H} 9 \mathrm{c} 2$ from hypoxia-induced apoptosis and restores oxidative balance by Akt-1-dependent manner. J Cardiovasc Pharmacol 2014; 64: 375-384.

$33 \mathrm{li}$ GWD, Force T. Protein kinase cascades in the regulation of cardiac hypertrophy. J Clin Invest 2005; 115: 527-537.

34 Tilton RG, Munsch CL, Sherwood SJ, Chen SJ, Chen YF, Wu C et al. Attenuation of pulmonary vascular hypertension and cardiac hypertrophy with sitaxsentan sodium, an orally active ET(A) receptor antagonist. Pulm Pharmacol Ther 2000; 13: 87-97.

35 Srinivasan GP, Delma CR, Elamaran A, Somasundaram T. ic effect of acetone extract of Curucma longa in Brine Shrimp, Artemia salina (L). Int $J$ Curr Biotechnol 2014; 2: 1-6.

36 Kolev TM, Velcheva EA, Stamboliyska BA, Spiteller M. DFT and experimental studies of the structure and vibrational spectra of curcumin. Int J Quantum Chem 2005; 102: 1069-1079.

37 Woldt E, Sebti Y, Solt LA, Duhem C, Lancel S, Eeckhoute J et al. Rev-erb- $\alpha$ modulates skeletal muscle oxidative capacity by regulating mitochondrial biogenesis and autophagy. Nat Med 2013; 19: 1039-1046.

38 Doggrell SA, Brown L. Rat models of hypertension, cardiac hypertrophy and failure. Cardiovasc Res 1998; 39: 89-105.

39 Gomez-Arroyo JG, Farkas L, Alhussaini AA, Farkas D, Kraskauskas D, Voelkel NF et al. The monocrotaline model of pulmonary hypertension in perspective. Am J Physiol Lung Cell Mol Physiol 2012; 302: L363-L369.

40 Aldashev A, Naeije R Problems of High Altitude Medicine and Biology: NATO Science for Peace and Security Series (Springer, 2006).

41 León-Velarde F, Maggiorini M, Reeves JT, Aldashev A, Asmus I, Bernardi L et al. Consensus statement on chronic and subacute high altitude diseases. High Alt Med Biol 2005; 6: 147-157. 
42 Tatarková Z, Kuka S, Račay P, Lehotský J, Dobrota D, Mištuna D et al. Effects of aging on activities of mitochondrial electron transport chain complexes and oxidative damage in rat heart. Physiol Res 2011; 60: 281-289.

43 Rosca MG, Vazquez EJ, Kerner J, Parland W, Chandler MP, Stanley W et al. Cardiac mitochondria in heart failure: decrease in respirasomes and oxidative phosphorylation. Cardiovasc Res 2008; 80: 30-39.

44 Folmes CDL, Dzeja PP, Nelson TJ, Terzic A. Mitochondria in control of cell fate. Circ Res 2012; 110: 526-529.

45 Baker RG, Hayden MS, Ghosh S, Liu Q, Chen Y, Auger-Messier M et al. Activation of NF-kappa B is required for hypertrophic growth of primary rat neonatal ventricular cardiomyocytes. Circ Res 2011; 13. 6668-6673. (c) (i)

This work is licensed under a Creative Commons Attribution 4.0 International License. The images or other third party material in this article are included in the article's Creative Commons license, unless indicated otherwise in the credit line; if the material is not included under the Creative Commons license, users will need to obtain permission from the license holder to reproduce the material. To view a copy of this license, visit http:// creativecommons.org/licenses/by/4.0/

(C) The Author(s) 2017

Supplementary Information accompanies the paper on Experimental \& Molecular Medicine website (http://www.nature.com/emm) 